\title{
Digital Holography of Second Harmonic Signal
}

\author{
Ye Pu and Demetri Psaltis \\ Department of Electrical Engineering, California Institute of Technology, Pasadena, CA 91125 \\ Phone: (626)395-2232, Fax: (626) 568-8437,Email: yepu@sunoptics.caltech.edu
}

\begin{abstract}
We report holographic recording of second harmonic radiations emitted from nanocrystals under femtosecond laser excitation. Our results show great promise of this novel technique to provide four-dimensional images for highly dynamic micro- and nano-systems.

(C) 2006 Optical Society of America

OCIS Codes: (090.0090) Holography; (180.6900) Three-dimensional microscopy; (190.3970) Microparticle nonlinear optics; (090.1000) Aberration compensation;
\end{abstract}

\section{Introduction}

Nonlinear optical microscopy has gained increasing importance in recent years [1]. The finite scanning speed constrains these techniques to two dimensions. Attempts to extend these techniques into three spatial dimensions over time [four-dimensional (4D) imaging] [2] result in limited success at a great sacrifice of framing time. In a contrast, complex micro- and nano-systems (for example living cells) are often highly dynamic, requiring instantaneous, whole-field 3D imaging with fast framing time. Dynamic holographic recording of scattering by microparticles embedded in 3D media has recently been successfully demonstrated [3]. Direct extension of this technique into microscopy faces great difficulties due to severe background scattering. Here we report successful results in second harmonic (SH) holography with $\mathrm{SH}$ generating nanocrystals under femtosecond laser excitation, which will bridge the holographic 4D imaging technique into microscopy.

\section{Experimental Setup}

Figure 1 shows the experimental setup for the SH holography. Femtosecond laser pulses of 810 nm wavelength are split into two branches at the beamsplitter BS1. Approximately $1 \mathrm{~mJ}$ of energy is delivered to the sample containing randomly embedded $130 \mathrm{~nm} 3 \mathrm{C} \mathrm{SiC}$ nanocrystals (crystal structure $\bar{F} \overline{4} 3 \mathrm{~m}$ ). The pump intensity at the sample site is approximately $1 \times 10^{11} \mathrm{~W} / \mathrm{cm}^{2}$. After the pump energy at the fundamental frequency is rejected, the weak SH radiations form the nanocrystals (the object wave) are collected by an aspheric lens (N.A. $=0.5)$ at magnification of $25 \times$. The rest of the pulse energy was sent to a BBO frequency doubler to obtain the reference SH wave. The object and reference waves are combined at the beamsplitter BS2, and the interference patterns are captured by the CCD camera. The optical path lengths of the two SH waves are carefully matched by the variable delay line.

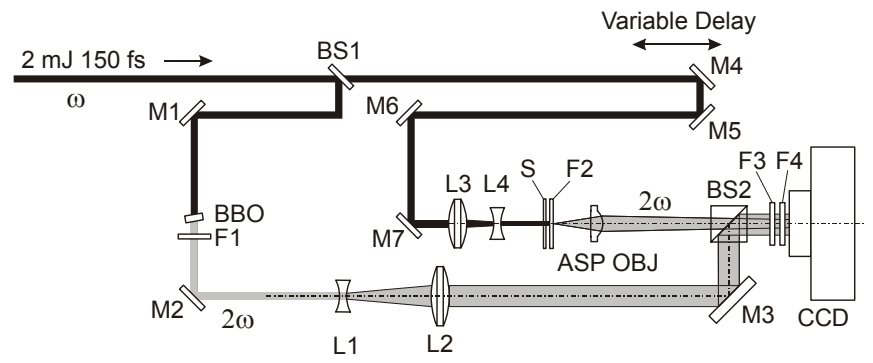

Figure 1. Experimental setup for second-harmonic holography. M1 - M7, mirrors; L1, L4, negative lenses; L3, L4, positive lenses; S, sample; F1 - F4, filters; BS1, BS2, beamsplitters; ASP OBJ, aspheric objective; BBO, beta barium borate crystal.

The digital image captured by the CCD camera is reconstructed numerically to obtain the electric field of the objective wave at the sample plane. The reconstruction algorithm is essentially a convolution between the holographic image intensity and a kernel function, which is obtained through ray tracing for a point source at certain object distance $z$ to compensate the severe spherical aberration.

\section{Results}

Figure 2 (a) shows a partial image of the SH hologram, which is captured at $10 \mathrm{~s}$ exposure time (integration of 100 pulses). Background spatial patterns due to dust on the optics are digitally subtracted. The general appearance of the fringes agrees with numerical simulation results for the same type of nanocrystals. Figure 2 (b) shows the reconstructed image of the nanocrystals. To the best of our knowledge, this is the first time holography is performed with SH scattering signals. 


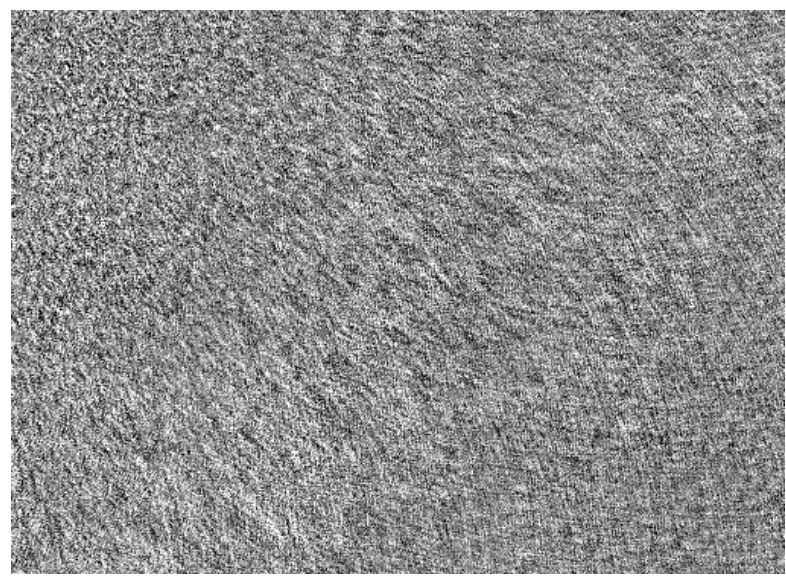

(a)

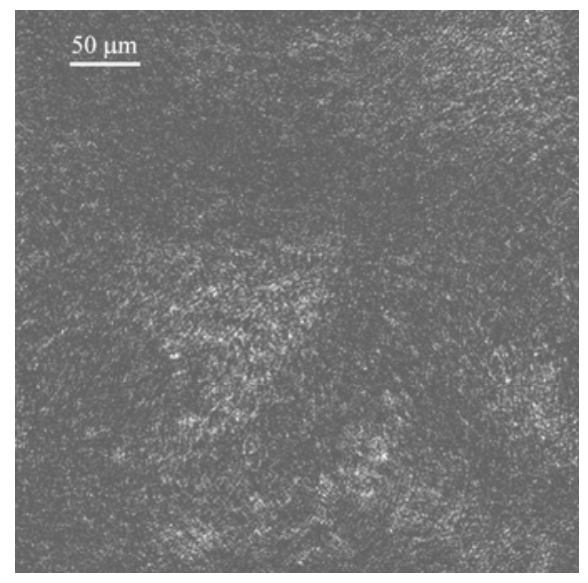

(b)

Figure 2. (a) Part of the SH holography fringes. Only a small portion of the hologram is shown to best reveal the holographic fringes. (b) The reconstructed image of the nanocrystals at the sample plane.

Zooming in on the reconstruction of individual nanocrystals at a series of distances $z$ reveals the axial intensity profile of single nanocrystal images, which is plotted in Figure 3. The transverse intensity profile of one particular nanocrystal image is also shown in the inset. Less than $1 \%$ of the background pixels have intensity exceeding the dotted line marked $1 \%$. On average, the transverse dimension of the reconstructed images is $1.6 \mu \mathrm{m}$, and the depth of focus is $1.75 \mu \mathrm{m}$. These values are very close to the diffraction limit. With larger N.A. these values can be improved to a submicron level. Furthermore, the center of each particle image can be located with subwavelength precision [4].

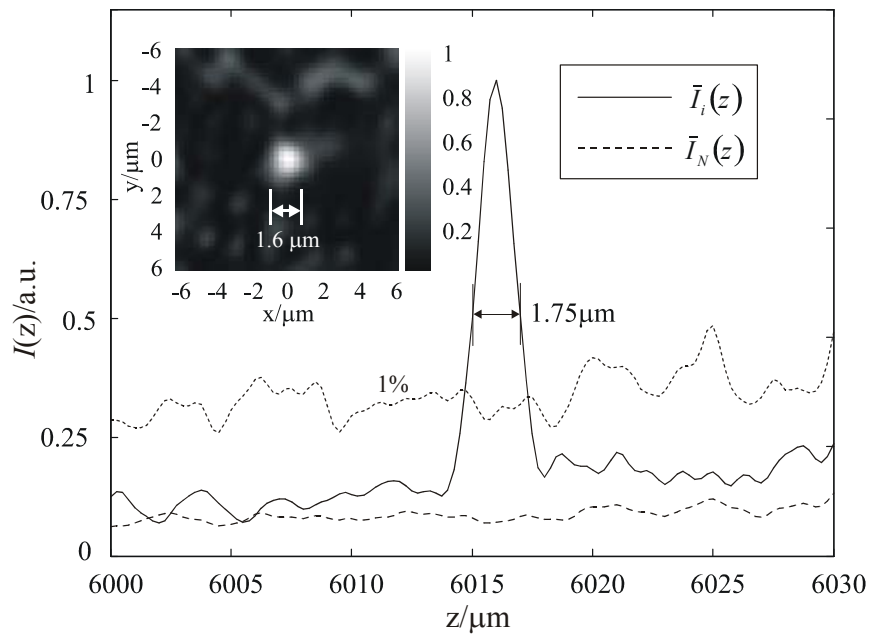

Figure 3. Mean axial intensity profile of the reconstructed particle images $\left[I_{i}(z)\right]$ and background $\left[I_{N}(z)\right]$. Totally 20 particles were picked out and their center intensity were scanned in axial direction to obtain $I_{i}(z) . I_{N}(z)$ were obtained in the same fashion for 100 random data points. The inset shows the transverse intensity profile of one particle image.

\section{Acknowledgement}

This work is supported by the DARPA Center for Optofluidic Integration at Caltech.

\section{References}

[1] W. R. Zipfel, R. M. Williams, and W. W. Webb, "Nonlinear magic: Multiphoton microscopy in the biosciences", Nature Biotechnol. 21, 1368-1376 (2003).

[2] D. Gerlich and J. Ellenberg, "4D imaging to assay complex dynamics in live specimens", Nature Cell Biol. 5, S14-S19 (2003).

[3] Y. Pu and H. Meng, "Four-dimensional dynamic flow measurement using holographic particle image velocimetry", to appear in Appl. Opt. (2005).

[4] J. Gelles, B. J. Schnapp, and M. P. Sheetz, “Tracking kinesin driven movements with nanometer scale precision", Nature 331, 450-453 (1988). 